This is a postprint version of the following published document:

Dios, M., et al. Semiconductor-metal core-shell nanostructures by colloidal heterocoagulation in aqueous medium. Materials Letters, October 2016, vol. 180, pp. 327-331 DOI: 10.1016/j.matlet.2016.05.179

(C) 2016 Elsevier

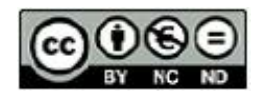

This work is licensed under a Creative Commons Attribution-NonCommercialNoDerivatives 4.0 International License. 


\title{
Semiconductor-Metal Core-Shell Nanostructures by Colloidal Heterocoagulation in aqueous medium
}

\author{
M. Dios ${ }^{a}$, Z. Gonzalez ${ }^{b, c,}$, E. Gordo ${ }^{a}$ and B. Ferrari ${ }^{b}$ \\ a) Department of Materials Science and Engineering and Chemical Engineering, University Carlos III, \\ Madrid, Spain. b) Institute for Ceramic and Glass, CSIC, Madrid, Spain. c) Hispano Italiana de \\ Revestimientos, S.A. Santander,Spain. \\ *corresponding author: zgonzalez@icv.csic.es
}

\begin{abstract}
In contrast to complex syntheses for the preparation of colloidal nanocomposites in a core-shell structure proposed in the literature, we present herein a facile colloidal route based on a heterocoagulation process promoted by the electrostatic interaction among ceramic NiO nanoplatelets and metallic Ni nanoparticles (NPs). Before the heterocoagulation process, $\mathrm{NiO}$ and Ni were synthetized separately in presence of ultrasound, by chemical precipitation and chemical reduction of the same nickel precursor, respectively. After that, NiO-Ni core-shell nanostructures were prepared forcing the electrostatic interaction among surfaces in aqueous medium. The surface charge balances of both types of particles were tuned effectively by adjusting the $\mathrm{pH}$ in a free-additives suspension. For the surface modification of $\mathrm{NiO}$ by $\mathrm{Ni}$, the ceramic suspensions maintain a positive zeta potential at $\mathrm{pH}$ 9, while the surface of metallic particles is negatively charged. Then the uniform coating of NiO platelets, by the electrostatically induced coagulation with Ni NPs, was favors. The degree of coverage and the formation of $\mathrm{NiO}-\mathrm{Ni}$ core-shell nanostructures were followed referring the evolution of zeta potential with the geometric calculation in terms of size and morphology of both nanoparticles, and then corroborated by field emission scanning electron microscopy (FESEM).
\end{abstract}

Keywords: Core-Shell, Heterocoagulation, Nickel Oxide, Metallic Nickel, Colloidal nanocomposites

\section{Introduction}

In the last decade, the scientific community has paid a special attention to nanocomposites based on semiconductor/metal structures in order to profit from the plasmonic effect of metallic nanocrystals in relevant fields of materials technology, such as energy storage, optoelectronics, photocatalysis or optical bioimaging [1]. Changes in the local environment of semiconductors promoted by the electric field enhancement near the surface of the metallic nanoparticles can significantly increase sensitivity of ceramic semiconductors. In this sense, large commercial applications of such hybrid nanostructures have generated the idea to incorporate an increasing number of nanoplasmonic and magnetoplasmonic metals (i.e Al, Fe, Co or $\mathrm{Ni}$ ) beyond $\mathrm{Au}$ and $\mathrm{Ag}$ [2-4]. Complementary, more recent studies show the relevance of the metal-semiconductor interface in the effectivity of the interfacial charge-transfer, and consequently tailoring of the heterostructure assembly to improve the electrochemical and/or catalytic response becomes new challenge $[2,4]$.

In the area of particle engineering, core-shell nanostructures with non-symmetrical shapes and 
compositions $[5,6]$ have been handled depending on the final application. Among all of them, inorganic/ inorganic core-shells have been proved to be extremely useful [5]. The combination of Nickel oxide (NiO) and Nickel (Ni) in a core-shell nanocomposite has already been proposed for many authors, mainly in the configuration of $\mathrm{Ni} / \mathrm{NiO}$ ( $\mathrm{Ni}$ as core and $\mathrm{NiO}$ as shell) due to its particular advantages in the electrochemical performance of devices for energy storage [7-9]. However the opposite configuration, oxide semiconductor $1 \mathrm{D}$ or $2 \mathrm{D}$ nanostructures decorated with $\mathrm{OD}$ metal nanoparticles, has paid a special attention because of the epitaxial growth of metal over the semiconductor nanostructure brings interesting plasmonic effects [1]. These semiconductor/metal hybrid nanostructures have been regularly fabricated by heteroprecipitation of the metal by the addition of a proper reducing agent. Moreover, the improved assembly of both nanostructures through an organic molecule has been successfully implemented. In this sense, this work proposes the assembly of Ni NPs onto NiO nanoplatelets by a direct heterocoagulation process. In a first step, both NiO and Ni NPs will be synthetized separately using two different routes, and later the core-shell assembly will be addressed when mixing both suspensions in the adequate ratio.

\section{Experimental procedure}

All chemicals were of reagent grade and used without any further purification. The synthesis and annealing of $\mathrm{NiO}$ nanoplatelets have been described elsewhere [10]. NiO was obtained from the calcination of as-synthesized $\beta-\mathrm{Ni}(\mathrm{OH})_{2}$ powder, prepared by chemical precipitation at room temperature using a high intensity ultrasonic horn $\left(45 \mathrm{~W} / \mathrm{cm}^{2}, 24 \mathrm{kHz}\right.$, titanium T13 tip, Sonopuls HD 2200, Bandelin Electronic, Germany). The thermal treatment was conducted in air, maintaining a dwell temperature of $325^{\circ} \mathrm{C}$ for $5 \mathrm{~min}$, with heating and cooling rates of $10^{\circ} \mathrm{C} / \mathrm{min}$.

Metallic Nickel nanoparticles (NP's) were synthesized by the chemical reduction of Nickel Nitrate Hexahydrate $\left(\mathrm{Ni}\left(\mathrm{NO}_{3}\right)_{2} \cdot 6 \mathrm{H}_{2} \mathrm{O}, 99,9 \%\right.$ purity, Panreac, Spain) with monohydrate Hydrazine $\left(\mathrm{N}_{2} \mathrm{H}_{4} \cdot \mathrm{H}_{2} \mathrm{O}\right.$, Sigma-Aldrich, Germany), also sonicating with the same ultrasonic horn (Sonopuls HD 2200). The synthesis procedure can be described as follow: a desired amount of Ni precursor was dissolved into deionized water (solution A). Separately, one mixture was prepared by dissolving Potassium Hydroxide $(\mathrm{KOH}$, Panreac, Spain) and monohydrate Hydrazine in water (mixture B). Then, mixture B was poured into solution A while US was applied. The temperature was controlled through the recirculation from a cryothermal bath. The products of synthesis were washed thoroughly for several times with deionized water at pH9 (adjusting with tetramethyl ammonium hydroxide, TMAH, Sigma-Aldrich, Germany) to residues removal. Ammonium Citrate tribasic $\left(\mathrm{HOC}\left(\mathrm{CO}_{2} \mathrm{NH}_{4}\right)\left(\mathrm{CH}_{2} \mathrm{CO}_{2} \mathrm{NH}_{4}\right)\right.$ Sigma-Aldrich, Germany) (CIT) was used in one case as synthesis modifier in a molar ratio $\left[\mathrm{Ni}^{2+}\right]:[\mathrm{CIT}]$ of 120:1. It was previously dissolved in the solution $A$ before the addition of the mixture $B$.

The studied variables have been: i) $\left[\mathrm{Ni}^{2+}\right]$ concentration (0.02M, 0.05M, 0.075M and $\left.0.1 \mathrm{M}\right)$, ii) $\mathrm{N}_{2} \mathrm{H}_{4} / \mathrm{Ni}^{2+}$ Ratio $(6,20,60)$, iii) Temperature $\left(40^{\circ} \mathrm{C}, 50^{\circ} \mathrm{C}\right)$, iv) Ultrasound Time (5 and 60 minutes), v) Ultrasound Power (100\% (63W) - 50\% (31.5W)) and vi) Synthesis modifier (CIT). 
Core-shell nanostructures preparation was carried out by inducing heterocoagulation of $\mathrm{Ni}$ and $\mathrm{NiO}$ suspensions. $1 \mathrm{~g} / \mathrm{L}$ and $10 \mathrm{~g} / \mathrm{L}$ suspensions of the calcined $\mathrm{NiO}$ and as-synthesized Ni nanopowders were prepared, respectively, at the tailored $\mathrm{pH}$ in deionized water. Different aliquots of the Ni suspension were added to a beaker where the $\mathrm{NiO}$ suspension was being beaten with a mechanical stirrer at 200 rpm. Later, suspensions were rolled overnight to promote the heterocoagulation between NiO nanoplatelets (with positive charge) and NP's (with negative charge).

The surface charge of NiO platelets and NPs was evaluated in terms of zeta potential. A Zetasizer Nano ZS (Malvern, UK) was used in order to determine the particle size distribution and zeta potential of all particles using Dynamic Light Scattering (DLS) and laser Doppler velocimetry, respectively. Suspensions used for determination were prepared with concentrations of $0.1 \mathrm{~g} / \mathrm{L}$ using $10^{-2} \mathrm{M} \mathrm{KCl}$ as solvent and inert electrolyte, so as to maintain the ionic strength of the medium. The $\mathrm{pH}$ adjustments of the suspensions were carried out by addition of small aliquots of $0.1 \mathrm{M} \mathrm{HNO}_{3}$ or $\mathrm{TMAH}$ and controlled with a $\mathrm{pH}$ probe (Metrohm AG, Germany). Subsequently, homogenization was achieved by sonication, using a UP400S Ultrasonic probe (Hielscher, Germany) for an optimized period of time.

Crystalline phases were characterized by X-Ray diffraction (Siemens-Bruker D8 Advance, Germany) using $\mathrm{Cu}$ Ka radiation ( $\lambda=1.540598$ Amstrongs). The diffraction patterns were measured step by step (0.05o in 2Ө). Finally, particle size, morphology and crystallography were also examined by field emission scanning electron microscopy (FE-SEM) in an S-4700 microscope (Hitachi, JAPAN) and high resolution transmission electron microscopy (HR-TEM using a Jeol JEM 2100 electron microscope operating at 200 kV and equipped with an Orius Gatan CCD Camera. To avoid degradation of the hydroxides, TEM images were recorded under low energy conditions.

\section{Results and Discussion}

The procedure for the obtaining of $\mathrm{NiO}$ nanoplatelets and their characterization has been described recently elsewhere [10]. Figure 1a shows the overall size of $\mathrm{NiO}$ nanoplatelets, aproximately between $150-200 \mathrm{~nm}$ in diameter and $10 \mathrm{~nm}$ in thickness. Crystallographically, peaks identified at the XRD diffractrogram (not shown) correspond to a face-centered cubic lattice (JCPDS Card file No 441159) with a unit cell dimension of $2.07 \AA$ and a crystallite size of $13.7 \mathrm{~nm}$ [11] calculated from the Scherrer equation.

NPs were synthesized by adding a mixture of monohydrated hydrazine/ $\mathrm{KOH}$ over the precursor of $\mathrm{Ni}^{2+}$, and imposing $\mathrm{pH}$ values higher than 10 , following the simplified equation:

$$
2 \mathrm{Ni}^{2+}+4 \mathrm{OH}^{-}+\mathrm{N}_{2} \mathrm{H}_{4} \rightarrow 2 \mathrm{Ni}+\mathrm{N}_{2}+4 \mathrm{H}_{2} \mathrm{O}
$$

Considering the oxidation-reduction potentials of these species, NPs can't be synthetized under pH 9.5 according to the Nerst equation [12]. Noting that our synthesis took place in presence of ultrasound, and as a consequence of the sonocrystallization the formation of numerous nuclei, homogeneously dispersed in the aqueous solvent, was expected[13]. It is known that ultrasonic treatment can lead to 
the formation of clusters of nanoparticles. However, in this work the ultrasound is mainly used to effectively disturb the precursor's solution and disperse the nuclei. Taking account these aspects, the influence of variables as ion nickel concentration, ultrasound time and the ultrasound power was evaluated in terms of the called "S parameter" $[\mathrm{W} /(\mathrm{mol} / \mathrm{L}) \cdot \mathrm{s}]$ which is equivalent to the number of watts per second that a mol/L of $\mathrm{Ni}^{2+}$ experiences in each synthesis. This parameter was defined to quantify the efficiency of each factor on the resulting size of the NPs $[13,14]$. .

All synthesis conditions are collected in table 1 and related to the process yield and the mean particle size of the NP's population, in number and volume. It is relevant to note that most of the NP's populations in volume exhibit bimodal distributions, where the distributions of a fine fraction of particles are centered between 70 and $345 \mathrm{~nm}$ and coarse agglomerates are in the range of 450-715 nm. The mean particle size of the fine fraction in volume fits the mean particle size in number. Consequently, those distributions evidence the NPs agglomeration during the synthesis. Firstly, the synthesis was driven by the formation of small primary particles, followed by the formation of large secondary aggregates. The partial agglomeration of Ni NPs can be due to their slight oxidation in the basic pH aqueous medium after the precipitation by reduction, and the later surface hydration which promotes the formation of soft links such as hydrogen bridges among nanoparticles.

All obtained X-Ray diffraction patterns of the synthesized NPs (not shown) determined by three characteristic peaks of the face-centered cubic structure of $\mathrm{Ni}\left(45^{\circ}, 52.4^{\circ}, 76.9^{\circ}\right)$, corresponding to Miller indices (111), (200) and (222) respectively according to the index card JCPDS no 04-0850. Besides, the pattern indicates the absence of impurities and secondary phases. The crystallite size of Ni NPs is 14.6 $\mathrm{nm}$ and the cell parameter is $3.48 \AA$. The unit cell dimension calculated from the Scherrer equation is higher than values reported for Ni NPs $(2.01 \AA)[2,15,16]$, while the crystallite size denotes that primary particles of 70-345 determined by DLS are made on nanocrystalline domains.

Although similar $\mathrm{Ni}$ nanocrystals were then the product of all the syntheses, in order to obtain the smallest population of Ni NPs, more relevant differences among particles in terms on DLS size were inspected. In table 1, when the S parameter decreases, either decreasing by half of the US power or increasing reaction time up to 60 minutes, the mean particle size of the NPs fine fraction increases from 90 to $300 \mathrm{~nm}$. All these syntheses started turning the solution's color to black, indicating that the reduction process was completely finished after the first $5 \mathrm{~min}$. Thus, the rest of the time was employed in increasing the size of the agglomerates (not desired).

Also, the results for the second variable, $\left[\mathrm{Ni}^{+2}\right]$, in table 1 indicate a decrease of the mean NPs size fine fraction from 105 to $80 \mathrm{~nm}$ due to the decrease of the $\mathrm{Ni}^{+2}$ concentration (keeping constant the T⿳a一, US power and reaction time). A reduction of Ni precursor leads to a higher effect of the US on the existing material and thus promotes the formation of smaller nuclei during the reduction process. Note that a threshold value (synthesis $\mathrm{Ni}-0.02 \mathrm{M}$ ) exists for the $\left[\mathrm{Ni}^{2+}\right]$ in which below $0.02 \mathrm{M}$ there is no reduction reaction, even after 60 minutes.

On the other hand, the decrease of $\mathrm{N}_{2} \mathrm{H}_{4}$ concentration (expressed as ratio between synthesis 
precursors) from 60, 20 to 6 (second and third variables studied in table 1), shows a slightly size growth of the NP's from 80 to $115 \mathrm{~nm}$ (keeping constant Ta, US power, reaction time and even [ $\left[\mathrm{Ni}^{2+}\right]$ ). In presence of a lower amount of reducing agent, only a few metallic Ni nuclei were formed in the early stage of the reduction reaction. Later, in successive steps, the growth of NP's is carried out through the Oswald Ripening [17] producing an increase of the agglomerates size. There is no formation of new nuclei. The reduction rate of the $\mathrm{Ni}^{2+}$ was slowed by diminishing the amount of hydrazine and a new threshold value for the synthesis Ni-6 was observed. The reaction did not occur below of a hydrazine ratio of 6 . Although the reducing agent was used in a supra-stoichiometric concentration, the selected values for all these experiments have always been lower than the values described previously by some authors in other works [12].

According to these results, Ni-2.1S synthesis was selected for the Ni NPs obtaining due to the high level of dispersion achieved if compare with the products of the syntheses Ni1.1S and Ni0.2S. Herein, the main NP's sizes (both in number and volume) were the smallest for the shortest reaction times. In addition, as one of the aims of this work is the preparation of the core-shell nanostructures where $\mathrm{Ni}$ size should be within the magnetoplasmonic ranges [2], a small amount of a citric salt was added as quelating agent to prevent the agglomerates growth in the aqueous solution $[18,19]$. Under this synthesis conditions a reduction of the NP's size was obtained. The distribution in volume of the fine fraction was centered in $67 \mathrm{~nm}$.

The plot in Figure $1 \mathrm{~b}$ shows the particle size distributions of NPs with Dv50 in the nanometric range $(<100 \mathrm{~nm})$, labeled in table 1 as Ni-CIT, Ni-0.05M y Ni-2.1S. These distributions verified that NPs form clusters in water with particle sizes ranging from ten to several hundred nanometers. Note that in the $\mathrm{Ni}-\mathrm{CIT}$ powder (considering the modifier addition) particle sizes ranged from 60 to $400 \mathrm{~nm}$ and 70 vol.\% of the particles were smaller than $80 \mathrm{~nm}$. By contrast, in the Ni-2.1S and Ni-0.05M powders (US time of $5 \mathrm{~min}$ and $0.05 \mathrm{M} \mathrm{Ni}^{2+}$ concentration, respectively) particle sizes ranged from 60 to 900-1200 $\mathrm{nm}$, and a lower volume of particles was smaller than $100 \mathrm{~nm}$. This result confirms that the addition of citrate maintains the NPs dispersed before the synthesis lowering the degree of agglomeration. The images in Figures $1 \mathrm{c}$ and $1 \mathrm{~d}$ show the Ni clusters and the dispersed NPs obtained from the synthesis with and without citrate, evidencing the nanometric size of the Ni NPs in both cases. 

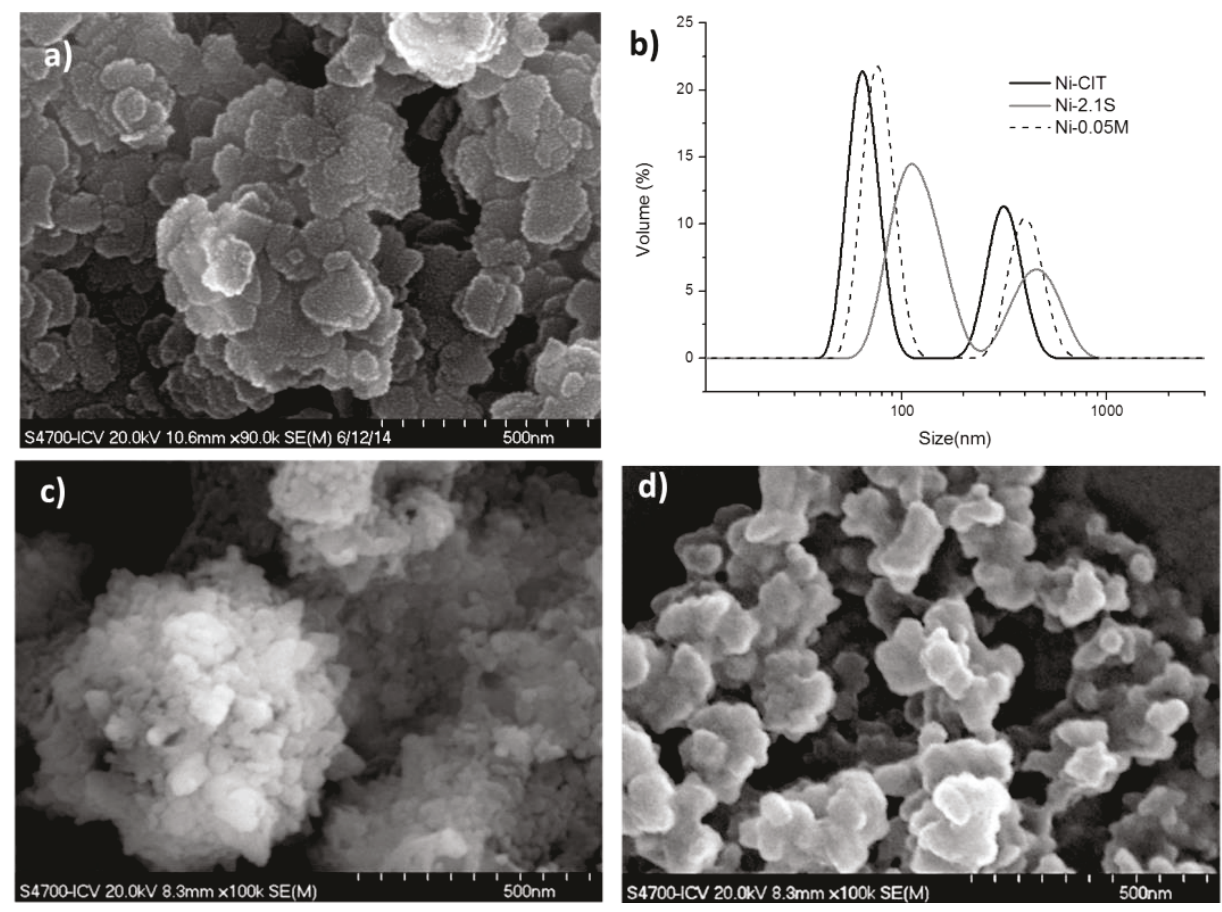

Figure1. a) FESEM image of NiO nanoplatelet, b)Particle size distribution of the syntheses Ni-CIT, Ni-2.1S and Ni-0.05M. And FESEM image of the clusters of Ni NPs synthesized without (c) and with (d) citrate.

The heterocoagulation between NiO nanoplatelets and NP's was carried out by direct contact of colloidal suspensions previously stabilized in aqueous medium. The colloidal stability of both suspensions was estimated from the zeta potential evolution with the $\mathrm{pH}$ (Figure 3a). Ni has an isoelectric point (IEP) at $\mathrm{pH}=5.5$ while the IEP of $\mathrm{NiO}$ is $\mathrm{pH}=12$. Maximum stability corresponds to $\mathrm{pH}$ ranges where zeta potential exhibits the higher absolute values, since the higher is this value the greater the repulsion between the colloidal particles of the same nature. NPs have two areas of stability at $\mathrm{pH}>7.5$ and $\mathrm{pH} 3$ while the $\mathrm{NiO}$ powder is stable from $\mathrm{pH}<10$. So, both powders are stable at $\mathrm{pH} 9$ but they have different sign of their surface charge, NiO has a zeta potential of $+35 \mathrm{mV}$, while the Ni NPs have $-55 \mathrm{mV}$. Therefore, this $\mathrm{pH}$ value was chosen in order to achieve the experimental procedure proposed.

The preparation of the core-shell nanostructures was carried out by inducing heterocoagulation in aqueous medium which consists on the assembly of both species throughout the electrostatic interactions developed among surfaces with opposite charge. So, the final material was obtained by direct contact of well-dispersed suspensions of $\mathrm{NiO}$ nanoplatelets and Ni NP's.

According to the size and morphology of both nanostructures, metal and semiconductor, the theoretical amount of NP's required to completely cover $(100 \%)$ the surface of the NiO nanoplatelets, was determined. To stablish the amount of NPs needed to cover $1 \mathrm{~g} / \mathrm{L}$ suspension of NiO nanoplatelets, an 
ellipsoidal cylinder with $150 \mathrm{~nm}$ in diameter and $10 \mathrm{~nm}$ in thickness was assumed for the NiO nanoplatelets, while Ni was considered spherical in shape with $60 \mathrm{~nm}$ in diameter.

Figure 2 shows the scheme of the core-shell. The volume occupied by $\mathrm{Ni}(\mathrm{Va})$ corresponds to the ring formed by the NPs surrounding the two faces of the NiO nanoplatelet (equation 2), where Vc is the volume of full core-shell structure and $\mathrm{Vb}$ is the volume of one $\mathrm{NiO}$ nanoplatelet.

$$
\mathbf{V}_{\mathbf{a}}+\mathbf{V}_{\mathbf{b}}=\mathbf{V}_{\mathbf{c}}
$$

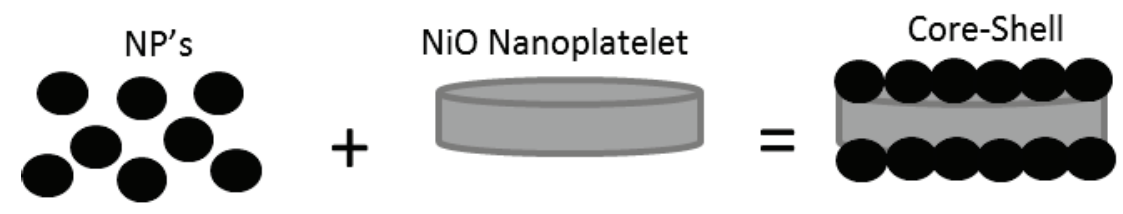

Figure 2. Diagrams of the formation of $\mathrm{NiO} / \mathrm{Ni}$ core-shell nanostructure

The number of NPs required to cover the nanoplatelets, results from the ratio of the volume of the shell (Va) and the volume of a Ni NPs (equation 3),

$$
\text { No of NP's }=\frac{\mathrm{Va}}{\mathrm{v}_{\mathrm{d}}}
$$

Considering the density of both materials $\left(6.67 \mathrm{~g} / \mathrm{cm}^{3}\right.$ and $8.91 \mathrm{~g} / \mathrm{cm}^{3}$ for $\mathrm{NiO}$ and $\mathrm{Ni}$ respectively), the amount of NPs needed to cover each NiO nanoplatelet was estimated. Following this protocol, different aliquots of the NPs as-synthesized suspension were added to the NiO suspension to cover a 10, 30, 50 and $100 \%$ of the NiO surface.
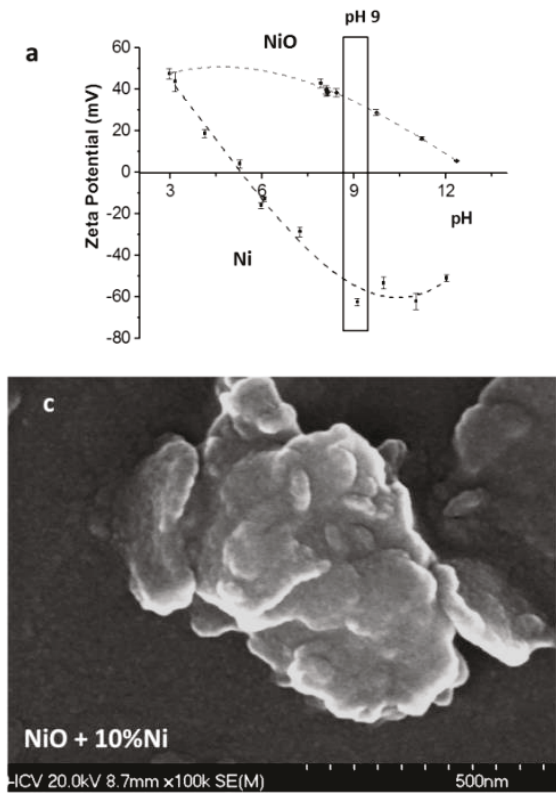

Figure 3. a) Zeta potential vs pH of NiO and NP's, b) Zeta potential vs Percentage of covering of the resulting Core-shell, c) and d) FESEM images of the $\mathrm{NiO}-\mathrm{Ni}$ particles covered at $\mathbf{1 0} \%$ and $\mathbf{5 0 \%}$ respectively 
The zeta potential of the resulting mixtures was determined and plotted in figure $3 \mathrm{~b}$. It shows an exponential decrease of the zeta potential values with the increase of the percentage of covering determined through the geometric calculus. That means, the surface charge balance changes from a positive value of $+26 \mathrm{mV}$ corresponding to the bare surface of the NiO (Figure $3 \mathrm{~b}$ ) to $-1,-11,-19$ and -22 $\mathrm{mV}$ for $10,30,50$ and $100 \%$ of covering respectively. Although the inescapable agglomeration of nanoplatelets during drying for SEM inspection, changes in the surface morphology of the particles, shown in figure $3 c$ and $3 d$, evidence the increase of percentage of covering of $\mathrm{Ni}$ over NiO from 10 to $50 \%$, by the heterocoagulation of both entities, and hence the core-shell formation.

\section{Conclusions}

$\mathrm{NiO} / \mathrm{Ni}$ core-shell nanocomposites have been successfully prepared in aqueous medium by a forced assembly process according to the dissimilar sign of their surface charges. We profit from the different colloidal behavior at $\mathrm{pH} 9$ in water, of the re-dispersed $\mathrm{NiO}$ nanoplatelets and as-synthesized $\mathrm{Ni}$ nanoparticles, to promote their assembly and produce semiconductor-metal nanocomposites. NiO coverage can be monitored by the zeta potential determination of the core-shell suspensions and confirmed by SEM.

Citrate plays a double role as synthesis modifier, extremely relevant to produce and maintain dispersed nanoparticles in aqueous media. The particle size of as-synthesized Ni decreases with the S parameter $[\mathrm{W} /(\mathrm{mol} / \mathrm{L}) \cdot \mathrm{s}]$, while the concentration of $\mathrm{Ni}^{2+}$ and the related amount of hydrazine has on each thresholds in order to produce Ni NPs under described US conditions. However, the suprastoichiometric concentration of hydrazine has been optimized in the reducing process of NP's.

\section{Acknowledges}

The authors acknowledge the support of the projects S2013/MIT-2862 and MAT2012-38650-C02-01, MAT2012-38650-C02-02. M. de Dios acknowledges MINECO through the grant FPI-2013 and Dr. Z González acknowledges to MINECO through the grant PTQ-13-05985.

\section{References}

[1] R. Jiang, B. Li, C. Fang, J. Wang, Metal/Semiconductor Hybrid Nanostructures for Plasmon-Enhanced Applications, Adv. Mater. 26 (2014) 5274-5309.

[2] D. Zeng, Y. Chen, Z. Wang, J. Wang, Q. Xie, D.-L. Peng, Synthesis of Ni-Au-ZnO ternary magnetic hybrid nanocrystals with enhanced photocatalytic activity, Nanoscale. 7 (2015) 11371-11378.

[3] Z. Pirzadeh, T. Pakizeh, V. Miljkovic, C. Langhammer, A. Dmitriev, Plasmon-Interband Coupling in Nickel Nanoantennas, ACS Photonics. 1 (2014) 158-162.

[4] Y. Chen, D. Zeng, M.B. Cortie, A. Dowd, H. Guo, J. Wang, et al., Seed-Induced Growth of Flower-Like Au-Ni-ZnO MetalSemiconductor Hybrid Nanocrystals for Photocatalytic Applications, Small. 11 (2015) 1460-1469.

[5] R.G. Chaudhuri, S. Paria, Core/Shell Nanoparticles: Classes, Properties, Synthesis Mechanisms, Characterization, and Applications, Chem. Rev. 112 (2012) 2373-2433.

[6] Q. Zhang, I. Lee, J.B. Joo, F. Zaera, Y.D. Yin, Core-Shell Nanostructured Catalysts, Acc. Chem. Res. 46 (2013) 1816-1824..

[7] J.H. Kim, S.H. Kang, K. Zhu, J.Y. Kim, N.R. Neale, A.J. Frank, Ni-NiO core-shell inverse opal electrodes for supercapacitors, Chem. Commun. 47 (2011) 5214-5216..

[8] A.C. Johnston-Peck, J.W. Wang, J.B. Tracy, Synthesis and Structural and Magnetic Characterization of Ni(Core)/NiO(Shell) Nanoparticles, ACS Nano. 3 (2009) 1077-1084..

[9] X.F. Li, A. Dhanabalan, C.L. Wang, Enhanced electrochemical performance of porous NiO-Ni nanocomposite anode for lithium ion batteries, J. Power Sources. 196 (2011) 9625-9630..

[10] Z. Gonzalez, B. Ferrari, A.J. Sanchez-Herencia, A. Caballero, J. Morales, Relevance of the Semiconductor Microstructure in 
the Pseudocapacitance of the Electrodes Fabricated by EPD of Binder-Free B-Ni(OH $)_{2}$ Nanoplatelets, J. Electrochem. Soc. 162 (2015) D1-D12.

[11] A. Caballero, L. Hernan, J. Morales, Z. Gonzalez, A.J. Sanchez-Herencia, B. Ferrari, A High-Capacity Anode for Lithium Batteries Consisting of Mesoporous NiO Nanoplatelets, Energy \& Fuels. 27 (2013) 5545-5551.

[12] J. Tientong, S. Garcia, C.R. Thurber, T.D. Golden, Synthesis of Nickel and Nickel Hydroxide Nanopowders by Simplified Chemical Reduction, J. Nanotechnol. 2014 (2014) 1-6.

[13] S. Cabanas-Polo, Z. Gonzalez, A.J. Sanchez-Herencia, B. Ferrari, Influence of ultrasound on the instantaneous synthesis of tridimensional [small alpha]- $\mathrm{Ni}(\mathrm{OH})_{2}$ nanostructures and derived NiO nanoparticles, CrystEngComm. 17 (2015) 61936206..

[14] M. Dios, Z. Gonzalez, E. Gordo, B. Ferrari, Core-shell Ti(C,N)-Ni structures fabricated by chemical precipitation of Nibased nanoparticles on TiCN suspensions, in: Euro PM, 2015.

[15] Y. Sahoo, Y. He, M.T. Swihart, S. Wang, H. Luo, E.P. Furlani, et al., An aerosol-mediated magnetic colloid: Study of nickel nanoparticles, J. Appl. Phys. 98 (2005).

[16] Y.Q. He, X.G. Li, M.T. Swihart, Laser-driven aerosol synthesis of nickel nanoparticles, Chem. Mater. 17 (2005) $1017-1026$.

[17] K.-M. Nam, H.-J. Kim, D.-H. Kang, Y.-S. Kim, S.-W. Song, Ammonia-free coprecipitation synthesis of a Ni-Co-Mn hydroxide precursor for high-performance battery cathode materials, Green Chem. 17 (2015) 1127-1135.

[18] L.Y. Wang, G.Q. Wu, D.G. Evans, Synthesis and characterization of a layered double hydroxide containing an intercalated nickel(II) citrate complex, Mater. Chem. Phys. 104 (2007) 133-140..

[19] K.S. Chou, K.C. Huang, Studies on the chemical synthesis of nanosized nickel powder and its stability, J. Nanoparticle Res. 3 (2001) 127-132..

\section{Figure Captions}

Figure1. a) FESEM image of NiO nanoplatelet, b) Particle size distributions of the syntheses $\mathrm{Ni}-\mathrm{ClT}, \mathrm{Ni}-$ $2.1 \mathrm{~S}$ and $\mathrm{Ni}-0.05 \mathrm{M}$. And FESEM image of the clusters of Ni NPs synthesized without (c) and with (d) citrate.

Figure2. Diagram of the formation of $\mathrm{NiO} / \mathrm{Ni}$ core-shell nanostructure

Figure3. a) Zeta potential vs $\mathrm{pH}$ of $\mathrm{NiO}$ and NP's, b) Zeta potential vs Percentage of covering of the resulting core-shell, c) and d) FESEM images of the $\mathrm{NiO}-\mathrm{Ni}$ particles covered at $10 \%$ and $50 \%$ respectively

Tables

\begin{tabular}{|c|c|c|c|c|c|c|c|c|c|c|c|}
\hline$\frac{\frac{0}{0}}{\frac{0}{\frac{\pi}{2}}}$ & $\begin{array}{c}\text { Molar ratio } \\
\left(\mathrm{Ni}^{2+}: \mathrm{KOH}: \mathrm{N}_{2} \mathrm{H}_{4}\right)\end{array}$ & $\begin{array}{c}\mathrm{T} \\
\left({ }^{\circ} \mathrm{C}\right)\end{array}$ & $\begin{array}{c}{\left[\mathrm{Ni}^{2+}\right]} \\
(\mathrm{M})\end{array}$ & $\begin{array}{l}\text { Time } \\
\text { (min) }\end{array}$ & $\begin{array}{l}P_{n} \\
(W)\end{array}$ & $\begin{array}{c}S \\
(W /(\mathrm{mol} / \mathrm{L}) \cdot \mathrm{s})\end{array}$ & $\begin{array}{l}\text { Yield } \\
(\%)\end{array}$ & $\begin{array}{l}\text { Dn50 } \\
\text { (nm) }\end{array}$ & \multicolumn{2}{|c|}{$\begin{array}{l}\text { Dv50 } \\
(\mathrm{nm})\end{array}$} & Labeled \\
\hline \multirow{3}{*}{$\mathbf{S}$} & \multirow{3}{*}{ 1:10:60 } & \multirow{3}{*}{50} & \multirow{3}{*}{0.1} & 5 & 63 & 2.1 & 84 & 104 & 120 & 461 & $\mathrm{Ni}-2.1 . \mathrm{S}$ \\
\hline & & & & 5 & 31.5 & 1.1 & 78 & 140 & 164 & 615 & $\mathrm{Ni}-1.1 \mathrm{~S}$ \\
\hline & & & & 60 & 63 & 0.2 & 83 & 300 & 342 & 615 & $\mathrm{Ni}-0.2 \mathrm{~S}$ \\
\hline \multirow{4}{*}[\mathrm{Ni}^{2+}]{} & 1:10:60 & \multirow{4}{*}{40} & 0.1 & \multirow{4}{*}{60} & \multirow{4}{*}{63} & 0.2 & 78 & 105 & 122 & 615 & $\mathrm{Ni0.1M}$ \\
\hline & 1:10:60 & & 0.075 & & & 0.2 & 84 & 90 & 91 & 531 & $\mathrm{Ni}-0.075 \mathrm{M}$ \\
\hline & $1: 10: 60$ & & 0.05 & & & 0.3 & 80 & 75 & 77 & 408 & $\mathrm{Ni}-0.05 \mathrm{M}$ \\
\hline & 1:10:60 & & 0.02 & & & 0.9 & - & - & - & - & $\mathrm{Ni}-0.02 \mathrm{M}$ \\
\hline \multirow{2}{*}[\mathrm{N}_{2}\mathrm{H}_{4}]{} & $1: 10: 20$ & \multirow{2}{*}{40} & \multirow{2}{*}{0.05} & \multirow{2}{*}{60} & \multirow{2}{*}{63} & \multirow{2}{*}{0.3} & 76 & 115 & 164 & 712 & $\mathrm{Ni}-20$ \\
\hline & $1: 10: 6$ & & & & & & - & - & - & - & $\mathrm{Ni}-6$ \\
\hline [CIT] & 1:10:60 & 50 & 0.1 & 5 & 63 & 2.1 & 85 & 64 & 67 & 323 & $\mathrm{Ni}-\mathrm{ClT}$ \\
\hline
\end{tabular}

Table 1 Synthesis conditions of Ni particles and results 